\title{
Entwicklung hochspezifischer und sensitiver Biosensoren auf Basis von bakteriellen Hüllproteinen und Aptameren
}

\author{
Ulrike Weinert $^{1)}$, Katrin Pollmann ${ }^{1)}$, Nadia Nikolaus ${ }^{2)}$, Beate Strehlitz $^{2)}$ und Johannes Raff ${ }^{1)}$ \\ 1) Helmholtz-Zentrum Dresden-Rossendorf e. V., Bautzner Landstraße 400, 01328 Dresden \\ ${ }^{2)}$ Helmholtz-Zentrum für Umweltforschung GmbH - UFZ, Permoserstr. 15, 04318 Leipzig
}

\section{Zusammenfassung}

Die vorliegende Arbeit befasst sich mit der Entwicklung nanoskaliger sensorischer Schichten basierend auf bakteriellen Hüllproteinen, Fluoreszenzfarbstoffen und Aptameren. Mittels der bakteriellen Hüllproteine soll eine Matrix erzeugt werden, die es erlaubt Aptamere und Fluoreszenzfarbstoffe mit wenigen Nanometern Abstand zueinander an eine Oberfläche, wie z. B. $\mathrm{SiO}_{2}$, zu binden. Dabei wirken die Aptamere als spezifische Rezeptoren für bestimmte Analyten und die Fluoreszenzfarbstoffe, welche in der Lage sind einen Fluoreszenz-Resonanz-Energie-Transfer (FRET) zu erzeugen, als optische Signalgeber. Die hier beschriebenen Ergebnisse zeigen, dass es möglich ist, Fluoreszenzfarbstoffe so an das Protein zu koppeln, dass ein Energietransfer entsteht. Auch konnte in ersten Versuchen gezeigt werden, dass die am Protein gekoppelten Aptamer funktional sind und in der Lage sind einen spezifischen Analyten zu binden.

\section{Einleitung}

Aufgrund immer genauerer Kontrollen von Lebensmitteln und Trinkwasser bezüglich Arzneimittelresten, wie z. B. Antibiotika, besteht ein großes Interesse an sensitiven und spezifischen Sensoren. Derzeit ist die Analyse von Lebensmitteln und Trinkwasser auf derartige Substanzen häufig mit einem großen Aufwand verbunden und kann meist nur offline erfolgen. Die Herausforderung besteht darin, Sensoren zu entwickeln, die sowohl eine schnelle Detektion ermöglichen, als auch einfach in ihrer Handhabung sind und spezifisch auf bestimmte Analyten reagieren.

Um diese Herausforderungen zu erfüllen, soll ein Sensor entwickelt werden, der sowohl spezifisch für einen Analyten ist, als auch eine schnelle Detektion über ein optisches Signal ermöglicht. Hierfür werden bakterielle Hüllproteine mit Aptameren und Fluoreszenzfarbstoffen miteinander gekoppelt.

Bakterielle Hüllproteine sind Strukturproteine, die die äußerste Membran von vielen Bakterien und fast allen Archaeen darstellen. Eine besondere Eigenschaft dieser Proteine ist, dass sie 2-dimensionale Gitterstrukturen ausbilden und nicht nur auf der Oberfläche von Bakterien oder Archaeen, sondern auch in wässrigen Systemen oder auf Oberflächen durch einen entropiegetriebenen Prozess zu Monolagen assemblieren. Weiterhin besitzen sie zahlreiche modifizierbare funktionelle Gruppen auf ihrer Oberfläche, welche sich besonders zur Bindung funktionaler Moleküle eignen. Die hier verwendeten bakteriellen Hüllproteine stammen von Bacillus-Stämmen, gewonnen aus Bodenproben der Uranabfallhalde "Haberland", Nähe Johanngeorgenstadt in Sachsen.

Für nachfolgenden Versuchsaufbau wurde das bakterielle Hüllprotein des Haldenisolates Lysinibacillus sphaericus JG-A12, welches eine p4 Symmetrie besitzt, verwendet. Die Gitterkonstante einer Einheitszelle, bestehend aus vier Monomeren des Proteins, ist mit etwa 13,5 nm im nanoskaligen Bereich, wodurch eine Miniaturisierung des Sensors möglich wird und auch die räumliche Nähe aller Komponenten zueinander gewährleistet ist. An die Oberfläche eines solchen Polymers werden nacheinander Fluoreszenzfarbstoffe und Aptamere kovalent gebunden.

Als Farbstoffkomponente werden zwei Fluoreszenzfarbstoffe verwendet, die in der Lage sind einen Fluoreszenz-Resonanz-Energie-Transfer (FRET) $\mathrm{zu}$ erzeugen. Dabei wird die Energie eines Donorfarbstoffes auf einen Akzeptorfarbstoff übertragen. Die zwei wichtigsten Vorraussetzungen sind dabei 
räumliche Nähe $(<10 \mathrm{~nm})$ und eine Überlappung des Donor-Emissionsspektrums mit dem AkzeptorAnregungsspektrums. Erkennbar wird der Energietransfer durch die Abnahme der Donoremission und gleichzeitige Zunahme der Akzeptoremission. Auch wird die Lebensdauer des Donormoleküls bei Anwesenheit eines Energietransfers gemindert, was über zeitaufgelöste Fluoreszenzmessungen sichtbar wird. Dieser beschriebene Energietransfer ist sehr empfindlich gegenüber störenden Molekülen. Bei Anwesenheit eines Akzeptors soll dieser Energietransfer daher gestört werden und die Änderung der Akzeptoremission bestimmt werden.

Als Rezeptor werden in der sensorischen Schicht Aptamere genutzt. Aptamere sind kurze Oligonukleotide, die über eine „Systematische Evolution von Liganden durch exponentielle Anreicherung“ (SELEX)-Prozess künstlich hergestellt werden und spezifisch einen Analyten über ihre 3D-Struktur nach dem SchlüsselSchloss-Prinzip binden. Sie werden daher auch chemische Antikörper genannt. Im Gegensatz zu Antikörpern sind sie jedoch äußerst stabil gegenüber äußeren Einflüssen und deutlich kleiner.

\section{Experimentelles}

\section{1 Modifizierung mit Fluoreszenzfarbstoffen und Messung des FRET}

Die verwendeten bakteriellen Hüllproteine wurden wie in [1] beschrieben von dem Haldenisolat Lysinibacillus sphaericus JG-A12 isoliert und als Polymersuspension für alle nachfolgenden Versuche verwendet.

Als FRET-Paar wurden die Fluoreszenzfarbstoffe HiLyte Fluor ${ }^{\mathrm{TM}} 488$ (H488) und HiLyte Fluor ${ }^{\mathrm{TM}} 555$ (H555) von der Firma Anaspec (Fremont, USA) verwendet, welche als Bindungsstelle eine $\mathrm{NH}_{2}$-Gruppe aufwiesen. Die Bindung der Fluoreszenzfarbstoffe erfolgte mittels 1-Ethyl-3-(3dimethylaminopropyl)carbodiimid (EDC) an die COOH-Gruppen des Proteins. Die Detektion des Energietransfers erfolgte sowohl über Fluoreszenzmessungen als auch über die Bestimmung der Lebensdauer des Donors. Für beide Messungen wurde das System QuantaMasterTM 40 - Q25 (Photon Technology International Inc., Birmingham, USA) verwendet.

\section{2 Modifizierung der bakteriellen Hüllproteine mit Anti-Thrombin-Aptamer (AT-Aptamer)}

Für die nachfolgenden Versuche wurde zunächst das gut untersuchte Anti-Thrombin Aptamer verwendet [2]. Dabei befand sich am 5'-Ende eine SH-Gruppe zur Kopplung des AT-Aptamers an das Protein und am 3'Ende der Fluoreszenzfarbstoff TAMRA, um den Erfolg der Bindung des Aptamers an das Protein leicht nachweisen und eventuell quantifizieren zu können. Als Crosslinker wurde PMPI eingesetzt, welcher SHGruppen mit $\mathrm{OH}-G r u p p e n$ verbindet. Die Funktionalität des gekoppelten Aptamers wurde mittels IAsysAffinitätssensor untersucht.

\section{Ergebnisse und Diskussion}

\section{2 Messung von FRET}

Der Nachweis des Energietransfers erfolgte sowohl über Fluoreszenzintensitätsmessungen als auch über zeitaufgelöste Fluoreszenzmessungen. Dabei lieferten bereits die Fluoreszenzintensitätsmessungen schon einen deutlichen Hinweis für einen Energietransfer. Für beide Messmethoden wurden Proben bakterielle Hüllproteine mit jeweils Donor oder Akzeptor als auch mit beiden Fluoreszenzfarbstoffen modifiziert In allen Proben wurde der Proteingehalt mittels Anregung bei $250 \mathrm{~nm}$ bestimmt. Damit konnte gewährleistet werden, dass sich in allen Proben eine etwa gleiche Menge Protein befindet. Die Ergebnisse sind in Bild 1 dargestellt. Zur Anregung des Donors H488 wurde eine Wellenlänge von $450 \mathrm{~nm}$ gewählt. Es ist erkennbar, dass bei dieser Anregungswellenlänge die Emission des nur mit Akzeptorfarbstoff modifizierten Proteins nahezu null ist. Auch bei dem Einsatz der doppelten Proteinmenge (vgl. Bild 1 linke Seite), ist keine Fluoreszenz erkennbar. Im Falle des nur mit dem Donor-Molekül modifizierten Proteins ist deutlich ein 


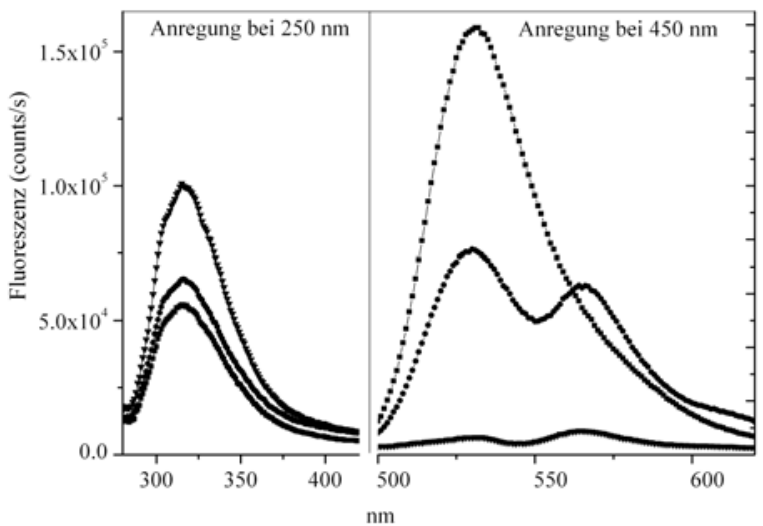

Bild 1 Emissionsspektren von bakteriellen Hüllproteinen modifiziert mit H488 (घ), H555 ( $\boldsymbol{\nabla}$ ) und beiden Fluoreszenzfarbstoffen (•); Mittels Anregung bei $250 \mathrm{~nm}$ wurden die Proteinmengen in den Proben quantifiziert und durch Anregung bei $450 \mathrm{~nm}$ wurde die Emission des Donors

H488 bestimmt. $\quad \operatorname{Im}$ Resultat erhält man eine Fluoreszenz-
zerfallskurve (siehe Bild 2), die einem exponentiellen Zerfall 1. Ordnung entspricht. Ebenfalls mit erfasst wird Impulsantwort, welche ca. 2 ns in der Peakbreite entspricht (siehe Bild 2).

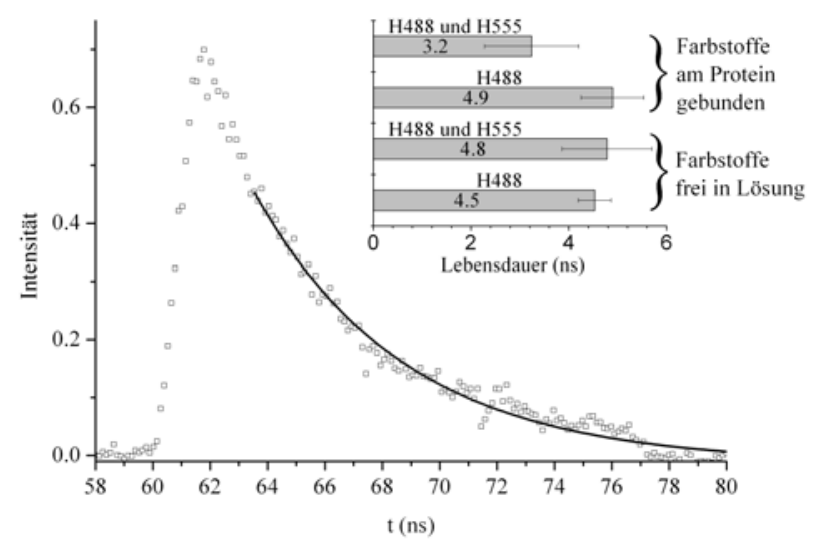

Bild 2 Fluoreszenzzerfallskurve des Donorfluoreszenzfarbstoffes H488, zusammen mit dem Akzeptorfloureszenzfarbstoff H555 am Protein gebunden; rechts oben sind die aus Fluoreszenzzerfallskurven berechneten Lebensdauern des Donors H488 dargestellt mit ihrer jeweiligen Standardabweichung aber nicht gebunden am Protein befinden. Die Kopplung der Fluoreszenzfarbstoffe an das Protein bewirkt eine hinreichend räumliche Nähe beider Fluoreszenzfarbstoffe zueinander und ermöglicht so den Energietransfer.

\section{2 Modifizierung von bakteriellen Hüllproteinen mit Aptameren}

Für die Modifizierung von bakteriellen Hüllproteinen mit Aptameren wurde zunächst Anti-ThrombinAptamer als Modellaptamer genutzt, da dieses Aptamer sehr gut charakterisiert und häufig in unterschiedlichen Assays als Modell verwendet wurde. Die Funktionalität des gekoppelten Aptamers zur Thrombin-Bindung wurde mittels resonant mirror spectroscopy mit dem System IAsys bestimmt. Hierfür wurden die mit AT-Aptameren modifizierten bakteriellen Hüllproteine als auch nicht modifizierte 
Hüllproteine an die Oberfläche der Messküvette kovalent über EDC gebunden. Die anschließende Messung beruht auf der Masseänderung der Sensorschicht innerhalb des evaneszenten Feldes der Küvettenoberfläche infolge der spezifischen Bindung des Liganden Thrombin an den immobilisierten Rezeptor AT-Aptamer und unspezifischer Anlagerungen auf der Küvettenoberfläche. Gemessen wird die Änderung des Resonanzwinkels (arc sec) des reflektierten eingestrahlten Lichts aufgrund der Änderung des Brechungsindex im Evaneszenzfeld.

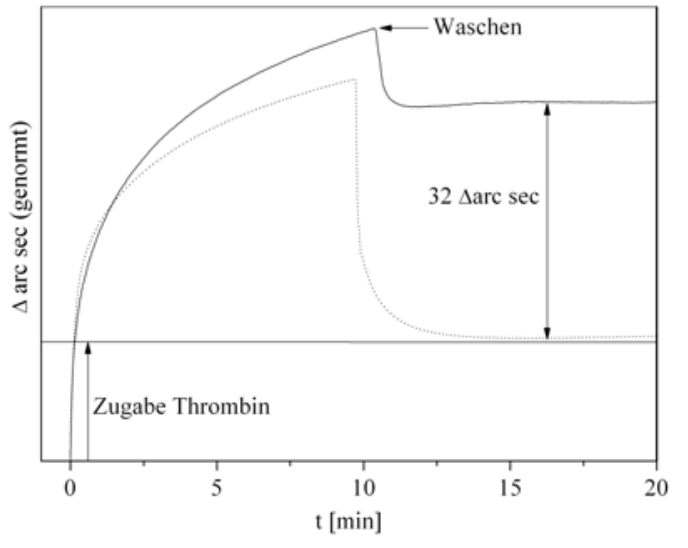

Bild 3 Chromatogramm von bakteriellen Hüllproteinen ohne Modifizierung ( (*) und mit AT-Aptamer gekoppelt (-).
Die Resonanzwinkeländerung wird über den Verlauf von Zugabe des Liganden, seine spezifische und unspezifische Anlagerung und einen Spülvorgang kontinuierlich gemessen. Dadurch ist eine Aussage möglich, inwieweit eine spezifische Bindung stattgefunden hat. In Bild 3 ist ein Vergleich zwischen mit AT-Aptamer gekoppelten bakteriellen Hüllproteinen und nicht modifizierten bakteriellen Hüllproteinen dargestellt. Nach Zugabe des Thrombins findet zunächst eine 10-minütige Sorptionsphase statt, in der sich das Thrombin spezifisch und unspezifisch an die modifizierte Küvettenoberfläche anlagert. Im Anschluss daran wird die Küvette gespült und unspezifisch gebundenes Thrombin entfernt. Im Diagramm ist deutlich erkennbar, dass bei der Probe mit nicht modifiziertem Protein keine Winkeländerung nach dem Waschen erkennbar ist. Wohingegen die mit modifizierten Protein beschichtete Küvette mit 32 arc sec eine deutliche Winkeländerung aufweist. Die Winkeländerung nach dem Waschschritt ist ein Maß für das spezifisch an das Aptamer gebundene Thrombin.

\section{Ausblick}

Es konnte gezeigt werden, dass sich die Einzelkomponenten, Fluoreszenzfarbstoffe und Aptamere, an die bakteriellen Hüllproteine binden lassen, ohne deren Struktur zu verändern. Auch konnte ein Energietransfer zwischen beiden Fluoreszenzfarbstoffen, gebunden an den bakteriellen Hüllproteinen, nachgewiesen werden. Ebenso zeigte sich, dass die Bindung von Aptameren an die bakteriellen Hüllproteine die Funktionalität des Aptamers nicht beeinträchtigt. In weiteren Versuchen sollen beide Komponenten auf dem Protein unter Funktionserhalt vereinigt werden. Durch Beschichtung entsprechender Oberflächen mit einer Hüllproteinschicht und Kopplung der verschiedenen Komponenten daran soll so eine sensorische Schicht erzeugt werden, die nach Bindung eines spezifischen Analyten ein verändertes Lichtsignal erzeugt und somit einen Nachweis des Analyten erlaubt. Ziel ist es eine Sensorschicht zu entwickeln, die spezifisch einen Arzneimittelstoff, wie z. B. Antibiotika, .in kleinsten Mengen im Wasser detektieren kann.

\section{Danksagung}

Diese Arbeit ist Bestandteil des Projektes „Aptamer modifizierte bakterielle Oberflächenstrukturen für die Entwicklung neuer Sensoren (AptaSens)“ und wird vom BMBF (BMBF/DLR 01RB0805A) unterstützt.

\section{Literatur}

[1] J. Raff et al., Chemistry of Materials 15 (1): 240-244, 2003.

[2] L. C. Bock et al., Nature (355): 564-566, 1992. 